\title{
Visible Light Photocatalytic Degradation of Methylene Blue and Malachite Green Dyes with $\mathrm{CuWO}_{4}$-GO Nano Composite
}

\author{
Sunitha Medidi1,2, Sowmyasree Markapurapu3 ${ }^{3}$, Mastan Rao Kotupalli4, \\ Rama Krishna Reddy Chinnam1, Venkata Mahalakshmi Susarla1, \\ Hima Bindu Gandham1, Paul Douglas Sanasi ${ }^{*}$ \\ ${ }^{1}$ Department of Engineering Chemistry, AU College of Engineering, Andhra University, Visakhapatnam, India \\ ${ }^{2}$ SKR College for Women, Rajamahendravaram, India \\ ${ }^{3}$ Centre for Nanotechnology, AU College of Engineering, Visakhapatnam, India \\ ${ }^{4}$ Department of Chemistry, University of Dodoma, Dodoma, Tanzania \\ Email: *spauldouglas.engchem@auvsp.edu.in
}

How to cite this paper: Medidi, S., Markapurapu, S., Rao Kotupalli, M., Chinnam, R.K.R., Susarla, V.M., Gandham, H.B. and Sanasi, P.D. (2018) Visible Light Photocatalytic Degradation of Methylene Blue and Malachite Green Dyes with $\mathrm{CuWO}_{4}$-GO Nano Composite. Modern Research in Catalysis, 7, 17-34.

https://doi.org/10.4236/mrc.2018.72002

Received: May 18, 2017

Accepted: April 27, 2018

Published: April 30, 2018

Copyright $\odot 2018$ by authors and Scientific Research Publishing Inc. This work is licensed under the Creative Commons Attribution International License (CC BY 4.0).

http://creativecommons.org/licenses/by/4.0/

\begin{abstract}
Copper Tungstate-Graphene Oxide nano composites have been successfully applied as excellent catalysts for the photocatalytic degradation with Methylene blue and Malachite green dyes under visible light irradiation. A facile solid state metathesis synthesis of copper tungstate $\left(\mathrm{CuWO}_{4}\right)$ followed by ball milling and subsequent preparation of copper tungstate-graphene oxide $\left(\mathrm{CuWO}_{4}-\mathrm{GO}\right)$ nano composite using a colloidal blending process and its application as a visible light photocatalyst for the degradation of Malachite green and Methylene blue dyes. The morphology and composition of copper tungstate $\left(\mathrm{CuWO}_{4}\right)$ nano composite have been characterized using X-Ray Diffraction (XRD), UV-Visible Diffuse Reflectance Spectra (UV-DRS), Raman Spectra, Field Emission Scanning Electron Microscopy (FESEM)-EDS and UV Visible Spectroscopy. It shows a band gap value of $2.13 \mathrm{eV}$, an increase in range and intensity of light absorption and the reduction of electron-hole pair recombination in $\mathrm{CuWO}_{4}$ with the introducing of $\mathrm{GO}$ on to it.
\end{abstract}

\section{Keywords}

Copper Tungstate, Graphene Oxide, Metathesis, Methylene Blue, Malachite Green, Visible Light Photodegradation

\section{Introduction}

Many efforts have been made in the past, and in recent years semiconductor 
based photocatalysts with high photocatalytic activity for environmental protection have been developed. Procedures such as water disinfection, air purification, polluted waste water remediation, etc. are taken. It has been a promising technique owing to its strong oxidizing nature, chemical inertness, economic viability and non-toxicity [1] [2] [3]. The unique arrangement of electronic structure, light absorption properties and charge transport characteristics in most of the metal oxides and tungstates have proven them to be superior photocatalysts.

Transition metal tungstates are considerable inorganic materials that have a significant application in various fields. Some of the divalent transition metal tungstates have also gained commercial interest in fluorescent lamps and lasers lights, due to its excellent electrical conductivity. In addition to this, these are also used as humidity sensors and catalysts. Copper tungstate is a well-known semiconductor with potential technological applications in scintillates, detectors, photon-odes, laser hosts, optical fibers etc. [4] [5].

Solid-state metathetic approach has been successfully applied for the synthesis of many oxide materials. For example, Gopalakrishnan et al. have synthesized oxides of $\mathrm{K}_{2} \mathrm{La}_{2} \mathrm{Ti}_{3} \mathrm{O}_{10}, \mathrm{Ca}_{2} \mathrm{La}_{2} \mathrm{CuTi}_{2} \mathrm{O}_{10}$ belonging to Ruddleson-Popper type of materials, $\mathrm{ABO}_{3}$ perovskite type of materials like $\mathrm{LaMO}_{3}(\mathrm{M}=\mathrm{Co}, \mathrm{Mn}), \mathrm{ATiO}_{3}$ $(\mathrm{A}=\mathrm{Ca}, \mathrm{Sr}$ and $\mathrm{Ba})$ and double perovskites like $\mathrm{Ba}_{3} \mathrm{MM}_{2} \mathrm{O}_{9}(\mathrm{M}=\mathrm{Mg}, \mathrm{Ni}, \mathrm{Zn}$; $\mathrm{M}_{-}=\mathrm{Nb}, \mathrm{Ta}$ ) by this approach [6] [7] [8] [9]. In addition, Kaner et al. have synthesized oxides of $\mathrm{Zr}$, Hf and $\mathrm{Cu}$ using this approach [10] [11]. Coppertungstate $\left(\mathrm{CuWO}_{4}\right)$ crystals exhibit only wolframite-type monoclinic structure at high pressure [12]. Many metal tungstates like $\mathrm{CuWO}_{4}$ are also used for water splitting and photocatalysis [13] [14].

Generally, the oxidized graphene sheets, namely, GO, acquire multiple defects and the degree of the defects is subject to the additive amount of oxidant and the oxidizing time [15]. Graphene oxide consists of water-dispersible, soft carbon sheets that can be easily converted to a conductive form and this 2D material continues to inspire many interesting applications and discoveries in a wide variety of fields including liquid-crystal display technology, bioscience, and materials science [16]-[21].

Graphene oxide (GO) is a two-dimensional material derived from graphite by introducing covalent $\mathrm{C}-\mathrm{O}$ bonds [22]. A large number of oxygen-containing functional groups have been implanted on both sides of a single graphite sheet overcomes the inter sheet Vander Waals force and enlarges the interlayer spacing. The sheets in such an expanded structure are easily pulled up using an external force by ultrasonication. Now the copper tungstate nano particles are directly grown on graphene oxide which appears to exhibit strong interactions with the underlying graphene oxide sheets. Since ultrasonication would not lead to any dissociation of the sheets, due to its strong coupling leading to an advanced hybrid materials for various applications including photocatalysis. The photo catalytic activity of the obtained copper tungstate-graphene oxide hetero-architecture was evaluated by the degradation of Malachite green (MG) and Methylene blue (MB) under visible-light, and the results are shown in Figure 1. 


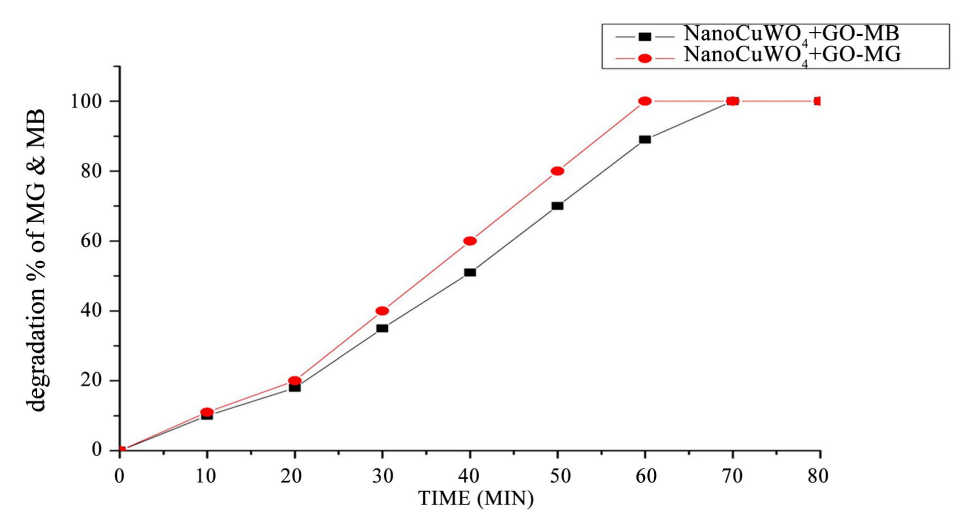

Figure 1. Photo degradation plots of Malachite green and methylene blue with copper tungstate, nano copper tungstate and nano copper tungstate-graphene oxide composite.

The reasons for these improvements are attributed to the fact that copper tungstate has a direct band gap positioned near the optical value of the solar spectrum and high energy of CB (Conduction Band), giving the photo electrons to a strong reducing ability and graphene oxide possesses a remarkable electrical transport property [23]. By this, the $\mathrm{CuWO}_{4}$-GO composite also possesses a remarkable electrical transport property. Now, in the $\mathrm{CuWO}_{4}-\mathrm{GO}$ composites, a possible reaction mechanism can be proposed based on the observed enhanced photo catalytic activity. Here, a portion of graphene oxide acts as a photo carrier in conducting electrons to the surface of the graphene oxide, which improves the separation of the electron-hole pairs and photo catalytic efficiency. It plays a conventional role as an elector acceptor and transporter, which is reported in most of the graphene oxide-semiconductor composites.

In the present study, a facile metathesis synthesis of $\mathrm{CuWO}_{4}$ and $\mathrm{CuWO}_{4}-\mathrm{GO}$ nano composite has been reported. The morphology and composition have been characterized using XRD, SEM and EDAX. The photo catalysis has been performed to study degradation of Methylene blue and Malachite green dyes by the prepared materials.

\section{Experimental}

\subsection{Materials and Methods}

All the chemicals were purchased of analytical grade which can be used as directly without any further purification. All the reactions were carried out using deionized water. The dyes used for this study are Malachite green and Methylene blue whose chemical formulae are $\mathrm{C}_{23} \mathrm{H}_{25} \mathrm{ClN}_{2}$ and $\mathrm{C}_{16} \mathrm{H}_{18} \mathrm{ClN}_{3} \mathrm{~S}$ respectively, and the stock solutions of the dyes were prepared in $10 \mathrm{ppm}$ concentrations in distilled water.

\subsection{General Procedure}

\subsubsection{Synthesis of $\mathrm{CuWO}_{4}$}

$\mathrm{CuWO}_{4}$ is prepared by solid-state metathesis synthesis followed by ball milling. 
Stoichiometric amounts of $\mathrm{CuCl}_{2}$ (LobaChemie Pvt. Ltd.) and $\mathrm{NaWO}_{4}(98 \%$ HIMEDIA) in the molar ratio of 1:1 are mixed in an agate mortar and grinded for about $4 \mathrm{hrs}$ in ethanol. With progressive grinding, the mixture exhibits a bright blue colour and the powder is calcinated at $400^{\circ} \mathrm{C}$ for 4 hours in a muffle furnace. After calcination, the compound is washed with distilled water several times to remove excess $\mathrm{NaCl}$ which is formed as a by-product and the residue is dried at $80^{\circ} \mathrm{C}$ in hot air oven and finally a greenish yellow copper tungstate powder is obtained which is then subjected to phase identification, micro-structural investigation and photocatalytic studies.

$$
\mathrm{CuCl}_{2} \cdot n \mathrm{H}_{2} \mathrm{O}+\mathrm{Na}_{2} \mathrm{WO}_{4} \rightarrow \mathrm{CuWO}_{4}+2 \mathrm{NaCl}+n \cdot \mathrm{H}_{2} \mathrm{O}
$$

The resultant compound $\mathrm{CuWO}_{4}$ obtained from the solid-state method was taken and grinded mechanically in low energy ball mill to obtain nano sized copper tungstate. The balls to powder weight ratio was maintained at 10:1 and the mixture is ground at $350 \mathrm{rpm}$ for $4 \mathrm{hrs}$ in methanol medium to obtain homogeneous compound. The residue after ball milling was dried in a hot air oven at $80^{\circ} \mathrm{C}$

\subsubsection{Synthesis of Graphene Oxide}

Graphene oxide (GO) was prepared by the well-known modified Hummers method [15] from an expanded acid washed graphite flakes (Sigma Aldrich). In this, $5 \mathrm{~g}$ of graphite was added into a mixture of $108 \mathrm{~mL}$ of sulphuric acid (Molychem-AR) and $12 \mathrm{~mL}$ of orthophosphoric acid (Molychem-AR) and stirred for $10 \mathrm{~min}$. To it, $2.5 \mathrm{~g}$ of sodium nitrate (Molychem-AR) was added. Subsequently, the beaker with reagents was put in an ice bath in order to keep it below $5^{\circ} \mathrm{C}$. Now, $15 \mathrm{~g}$ of potassium permanganate (Molychem-AR) was added in portions into the mixture, which was vigorously stirred. After addition of the oxidant, the beaker was heated and kept at $35^{\circ} \mathrm{C}$ to $40^{\circ} \mathrm{C}$ with continuous stirring. In the next step, $280 \mathrm{~mL}$ of deionized water was added to the beaker and heated to $95^{\circ} \mathrm{C}$ and maintained the same conditions for about $60 \mathrm{~min}$. To complete the reaction $5 \mathrm{ml}$ of $30 \%$ hydrogen peroxide (Molychem-AR) is added, now the color of the solution changes to bright yellow. The mixture is then washed with $5 \%$ hydrochloric acid (Molychem-AR) solution to remove sulfate ions and with deionized water in order to remove the chloride ions to maintain neutral $\mathrm{pH}$. After centrifugation the gel like substance is vacuum dried at $60^{\circ} \mathrm{C}$ for more than $6 \mathrm{hrs}$ to get $\mathrm{GO}$ as powder.

\subsubsection{Synthesis of $\mathrm{CuWO}_{4}$-GO Nano Composites}

$\mathrm{CuWO}_{4}$-GO nano composites were synthesized using a colloidal blending process with $\mathrm{GO}$ and $\mathrm{CuWO}_{4}$. For the preparation of $\mathrm{CuWO}_{4-} 10 \% \mathrm{GO}$ nano composites, $1 \mathrm{ml}$ of $10 \%$ GO was dispersed in $20 \mathrm{~mL}$ of deionized water and sonicated using ultrasonic cleaner for 30 minutes and $1 \mathrm{gm}$ of copper tungstate is dispersed in the prepared GO solution using sonicator for another 30 minutes. The above mixture is under constant stirring for about 4 hours. The bluish yellow product was collected and dried in hot air oven at $80^{\circ} \mathrm{C}$ for 2 hours [24]. Si- 
milarly $1 \%, 2 \%, 3 \%, 5 \%$ and $20 \%$ GO nano composites are prepared and used.

The obtained GO-CuWO${ }_{4}$ composite was characterized by X-Ray Diffraction (XRD), FT-IR, Scanning Electron Microscopy (SEM) and EDS, UV-Diffused Reflectance spectroscopy, Raman Spectroscopy.

\subsubsection{PhotoCatalytic Experiments}

Photo catalytic activity of the synthesized $\mathrm{CuWO}_{4}$-GO nano composite was evaluated by de-colorization of Methylene blue and Malachite green dye solutions. The experiments were carried out under the visible light irradiation in presences of $\mathrm{CuWO}_{4}-\mathrm{GO}$ photocatalyst. The photocatalysis process is carried out in a visible light photo reactor constructed with an outer wooden cabinet equipped with a magnetic stirrer, a Metal Halide Lamp, an exhaust fan and electric power supply cable. The reaction was carried out by adding $0.05 \mathrm{~g}$ of the as-synthesized nano composite into $100 \mathrm{~mL}$ of dye solution (10 ppm) in a $1000 \mathrm{~mL}$ Borosil glass beaker. The suspension was magnetically stirred in dark for 30 minutes to obtain desorption/absorption equilibrium before irradiating the solution to the light. A $400 \mathrm{~W}$ metal halide lamp is used as light source for the photo catalytic studies. The solution was irradiated and aliquots were drawn at regular time intervals, centrifuged and the transparent dye solution analyzed for absorbance using Visible Spectrophotometer ( $340 \mathrm{~nm}$ to $960 \mathrm{~nm}$ ).

Percentage degradation of the dye was calculated using the following formula.

$$
\text { \%Degradation }=\left(A_{o}-A_{t}\right) / A_{o}
$$

where $A_{o}$ is absorbance of dye at initial stage $A_{t}$ is absorbance of dye at time $t$.

\subsubsection{Instrumentation}

Retsch $^{\circledast}$ Planetary Ball Mill PM 100 bench top grinding station has been used for ball milling. The resulting powder was characterized using X-Ray Diffractometer (PANalytical-X' Pert PRO, Japan) at room temperature, using Nickel Filter $\mathrm{Cu}$-Ka radiation $(\lambda=1.54059 \AA)$, over a wide range of $10^{\circ} \leq 2 \theta \leq 80^{\circ}$ with a scanning speed of $2^{\circ} \mathrm{min}^{-1}$. The morphology of the as-synthesized samples was investigated by field emission scanning electron microscopy (FESEM, LEO1550). Band gaps were calculated using Single Monochromator UV-2600 (optional ISR-2600 Plus, $\lambda$ up to $1400 \mathrm{~nm}$ ).

\section{Results and Discussion}

\subsection{X-Ray Diffraction}

Figure 2 shows the X-Ray Diffraction plot of nanographene oxide and the peaks are in good agreement with the characteristic peaks of graphene oxide Figure 3 shows the X-Ray Diffraction plot of $\mathrm{CuWO}_{4}-\mathrm{GO}$ nano composite. The peaks of the composite are in good agreement with the characteristic peaks of $\mathrm{CuWO}_{4}$. While a distinct peak for pure graphene oxide was observed at $2 \theta=10.2^{\circ}$ (Figure 2), this GO related peak is not observed in $\mathrm{CuWO}_{4}-\mathrm{GO}$ plot due to the low amounts of GO and the resultant low diffraction intensity that would be 


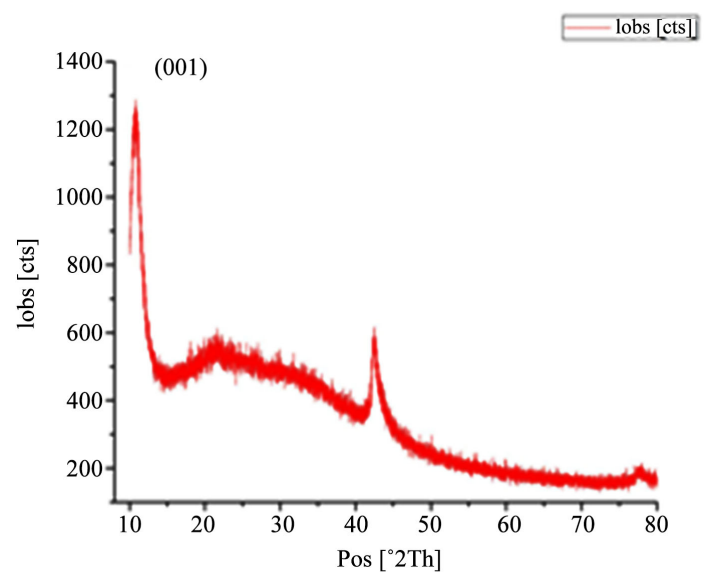

Figure 2. XRD-Spectrum of graphene oxide.

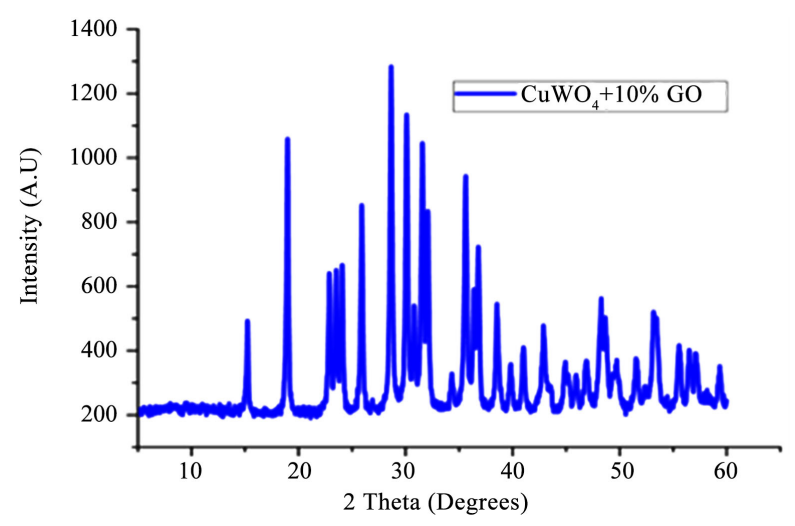

Figure 3. XRD-Spectrum of CuWO4-GO nano composite.

below the limit of detection of the instrument [23]. The phase purity of the as-synthesized materials was confirmed by representative XRD analysis. As shown in Figure 3 as-prepared powder samples have similar narrow characteristic peaks locating at $15.1^{\circ}, 19.2^{\circ}, 23.8^{\circ}, 24.9^{\circ}, 29.2^{\circ}, 31.6^{\circ}, 36.7^{\circ}$ and $43.8^{\circ}$, which match well with the (010), (001), (110), (0 11), (111), (111), (200) and (121) planes, respectively. All the mentioned crystal planes are in good agreement with the standard patterns for triclinic phase $\mathrm{CuWO}_{4}$ (JCPDS No 80-1918) [25].

\subsection{FT-IR}

The FTIR spectrum of $\mathrm{CuWO}_{4}$ is shown in Figure 4 The spectrum exhibits a broad band near $3446 \mathrm{~cm}^{-1}$ due to the $\mathrm{OH}$-stretching vibrations of free and hydrogen-bonded hydroxyl groups. The band at $614 \mathrm{~cm}^{-1}$ and the peak at $476 \mathrm{~cm}^{-1}$ can be assigned to the $\mathrm{Cu}-\mathrm{O}$ stretching vibration along the direction and the peak at $1028 \mathrm{~cm}^{-1}$ is due the presence of W-OH bond [26].

\subsection{Composition and Morphology Study}

Figure 5 shows the FE-SEM of $\mathrm{CuWO}_{4}$-GO nano composite. The nano structures and morphologies of samples are examined by SEM characterization. It shows the morphology of $\mathrm{CuWO}_{4}$-GO and it obviously reveals that micro structured 


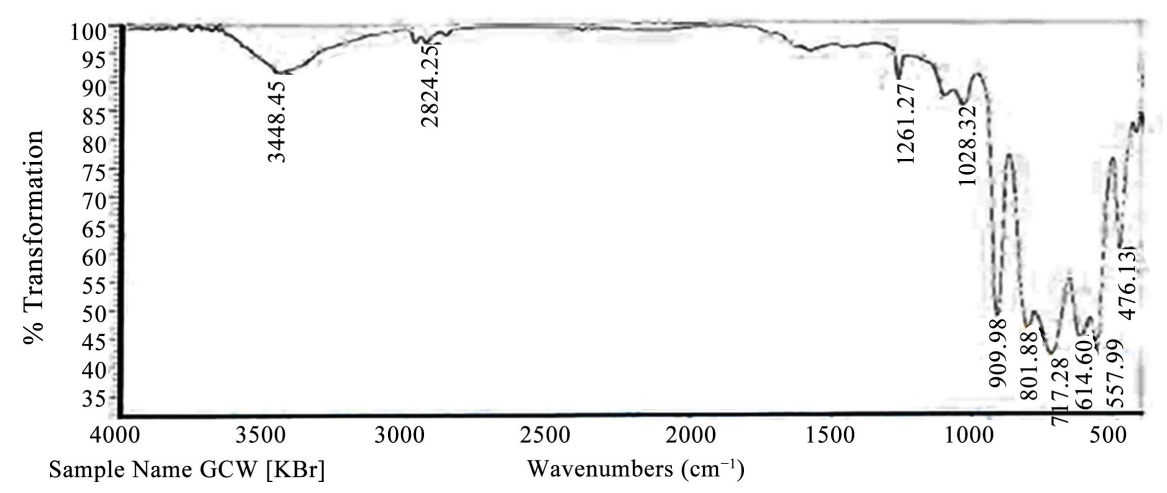

Figure 4. FTIR spectrum of $\mathrm{CuWO}_{4}$.
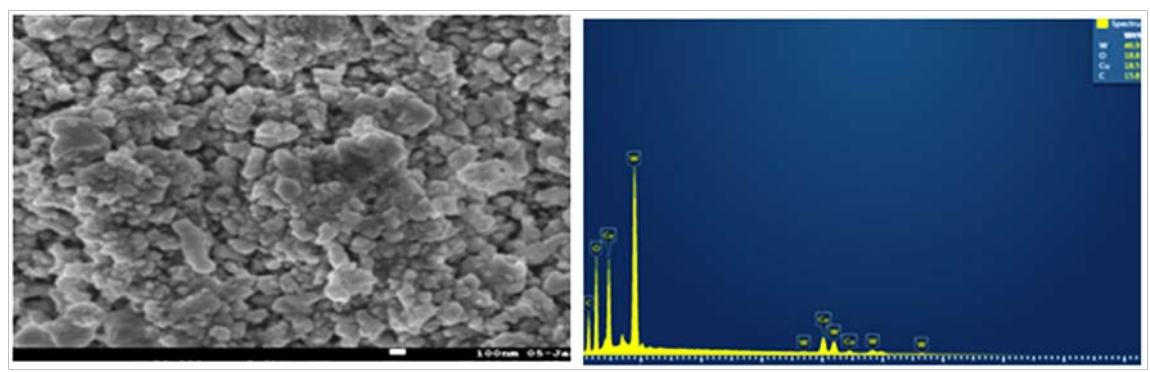

Figure 5. SEM-micrographs of $\mathrm{CuWO}_{4}$-GO nano composite and EDS spectra.

$\mathrm{CuWO}_{4}$ particles have a rough surface, with various irregular particles anchored on the surface of $\mathrm{CuWO}_{4}$. From this, it can be clearly observed that the prepared sample consists of nano particles in the size of $<50 \mathrm{~nm}$ in diameter. Further, the EDS spectrum and elemental composition demonstrates the presence of $\mathrm{Cu}, \mathrm{W}$, $\mathrm{O}$ and $\mathrm{C}$ which confirms the proper formation of $\mathrm{CuWO}_{4}$ - $\mathrm{GO}$ nano composite. EDS analysis shows a composition of $46.9 \% \mathrm{~W}, 18.8 \% \mathrm{O}, 18.5 \% \mathrm{Cu}$ and $15.8 \% \mathrm{C}$ (wt.\%) as evidence of successful inter-diffusion and the formation of $\mathrm{Cu}$ $\mathrm{WO}_{4}-\mathrm{GO}$ nano composite.

\subsection{UV Diffuse Reflectance Spectral Studies}

The optical properties of pure copper tungstate, nano copper tungstate and $\mathrm{CuWO}_{4}$-GO nano composite was evaluated by UV-Diffuse Reflectance Spectroscopy as shown Figures 6-9. Micro sized copper tungstate shows the $\lambda_{\max }$ at $290 \mathrm{~nm}$ and the calculated band gap was found to be $4.5 \mathrm{eV}$ (Figure 6). The nano sized copper tungstate shows a $\lambda_{\max }$ at $580 \mathrm{~nm}$ and the band gap has been reduced to $2.1 \mathrm{eV}$ (Figure 7). A steep decrease in the band gap of nanocopper tungstate indicates that the absorption in the visible light region is possible from a band gap transition from macro size to nano size. Specifically, electrons on the valence band were activated by photon energy and jumped to the conduction band [27]. By introducing graphene oxide on the surface of nano copper tungstate, a similar absorption edge was observed indicating that the graphene oxide was not incorporated into the lattice of copper tungstate and just got adsorbed on its surface. From the absorbance spectra for $\mathrm{CuWO}_{4}$-GO nano composite 


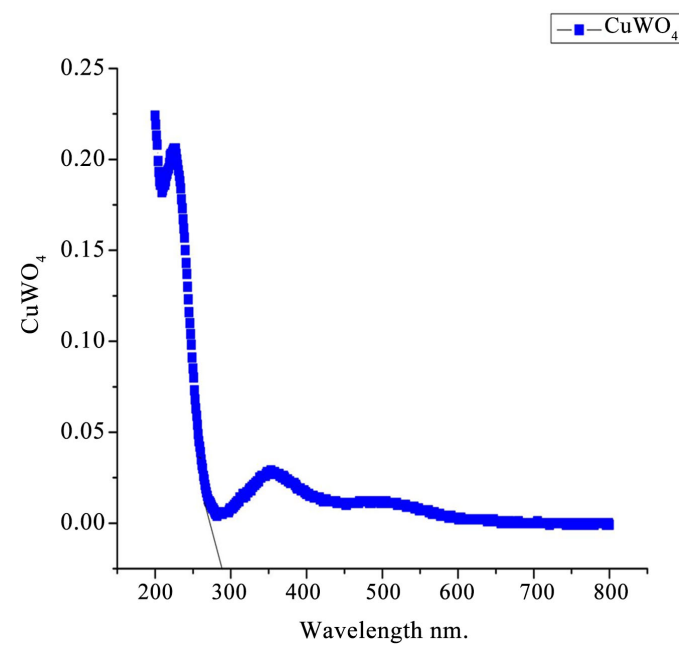

Figure 6. Band gap spectrum of $\mathrm{CuWO}_{4}$ (prepared by metathesis).

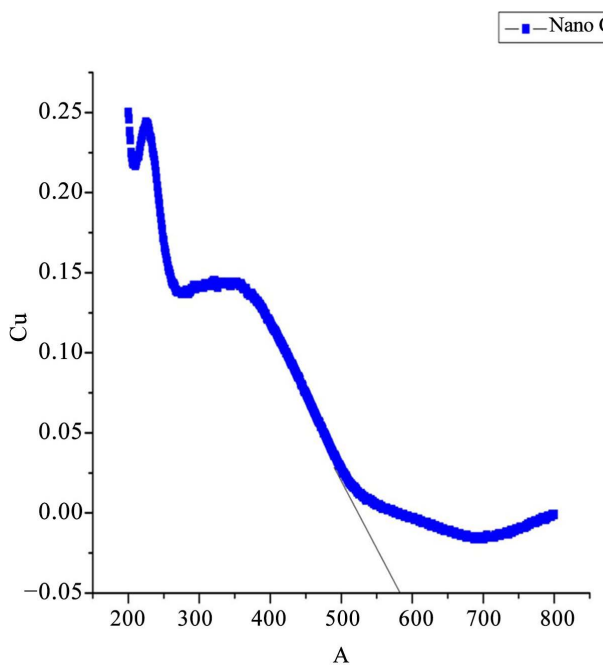

Figure 7. Band gap spectrum of $\mathrm{CuWO}_{4}$ (after low energy ball milling).

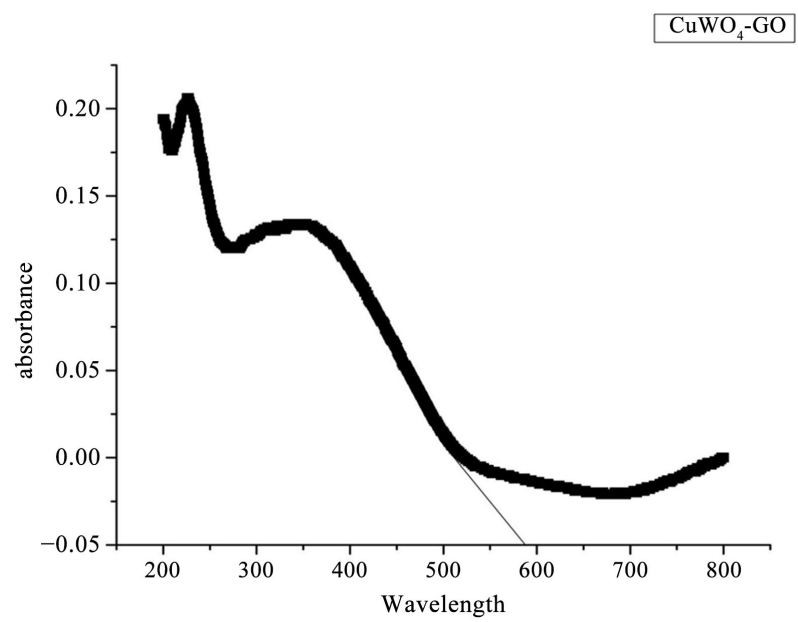

Figure 8. Band gap spectrum of $\mathrm{CuWO}_{4}-\mathrm{GO}$ composite. 


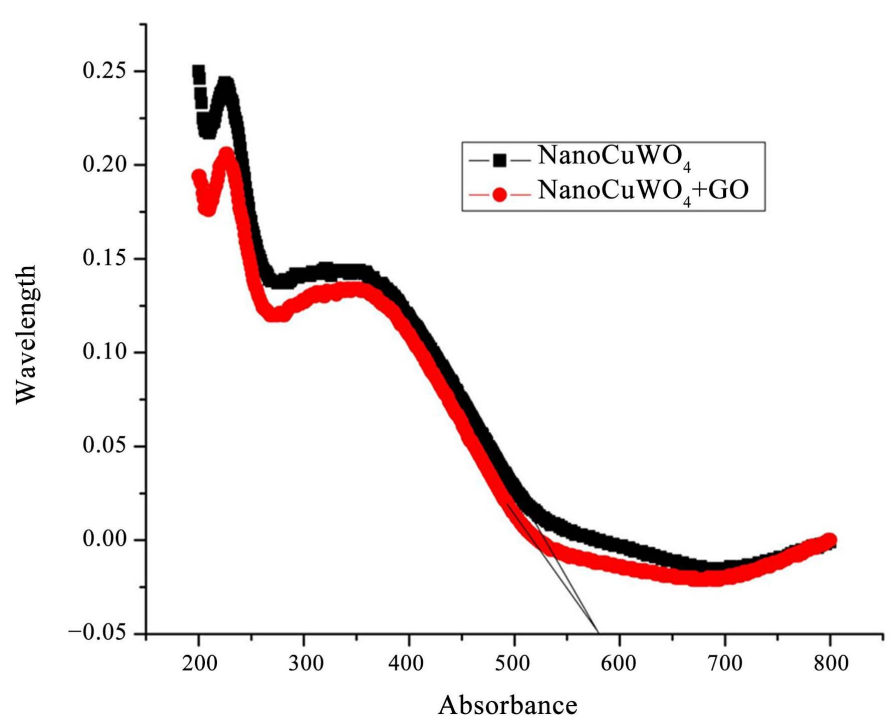

Figure 9. Band gap overlay spectrum of $\mathrm{CuWO}_{4}, \mathrm{CuWO}_{4}-\mathrm{GO}$.

the $\lambda_{\max }$ is observed at $580 \mathrm{~nm}$ and the band gap is found to be $2.1 \mathrm{eV}$ in Figure 8 and Figure 9.

The band gap of a solid and the wavelength of light that is reflected by it are related to one another via the Planck-Einstein Relation, which is stated below. $\mathrm{E}$ is the gap energy of the band gap in joules, $\mathrm{h}$ is Planck's constant $\left(6.63 \times 10^{-34} \mathrm{~J}\right.$ s), $v$ is frequency in hertz.

$$
E_{g}=h v=\frac{h c}{\lambda}
$$

From the plot of absorbance vs wavelength $(\lambda)$, wavelength of the material is measured and band gap is calculated from the equation. Where $\lambda$ is the wavelength of the material from the graph. $E_{g}$ is the bandwidth to be calculated

$$
E_{g}=1240 / \lambda(\mathrm{nm})
$$

\subsection{Raman Spectral Analysis}

Figure 10 shows Raman spectra of $\mathrm{CuWO}_{4}$-GO nano composite. In terms of group theoretical analysis, Wolframite structure belonging to $\mathrm{P}_{2} / \mathrm{c}(\mathrm{z}=2)$ monoclinic structure is expected to give $18\left(8 \mathrm{~A}_{\mathrm{g}}+10 \mathrm{~B}_{\mathrm{g}}\right)$ Raman-active bands out of 36 possible lattice modes. As our interest is limited to show the phase formation of tungstates, the Raman modes $\mathrm{CuWO}_{4}-\mathrm{GO}$ is shows the vibrations at 910 $\mathrm{cm}^{-1}$ which is an evidence for wolframite structure.

\subsection{UV-Visible Spectroscopy}

Figure 11 and Figure 12 show that high intensity peak at $667 \mathrm{~nm}$ for Methylene blue and $617 \mathrm{~nm}$ for a Malachite green dyes. The absorption wavelength of Methylene blue was recorded at $667 \mathrm{~nm}$ and Malachite green was recorded at 617 $\mathrm{nm}$ throughout the study and the percentage of degradation of Methylene blue and Malachite green was carefully monitored at various time intervals. 


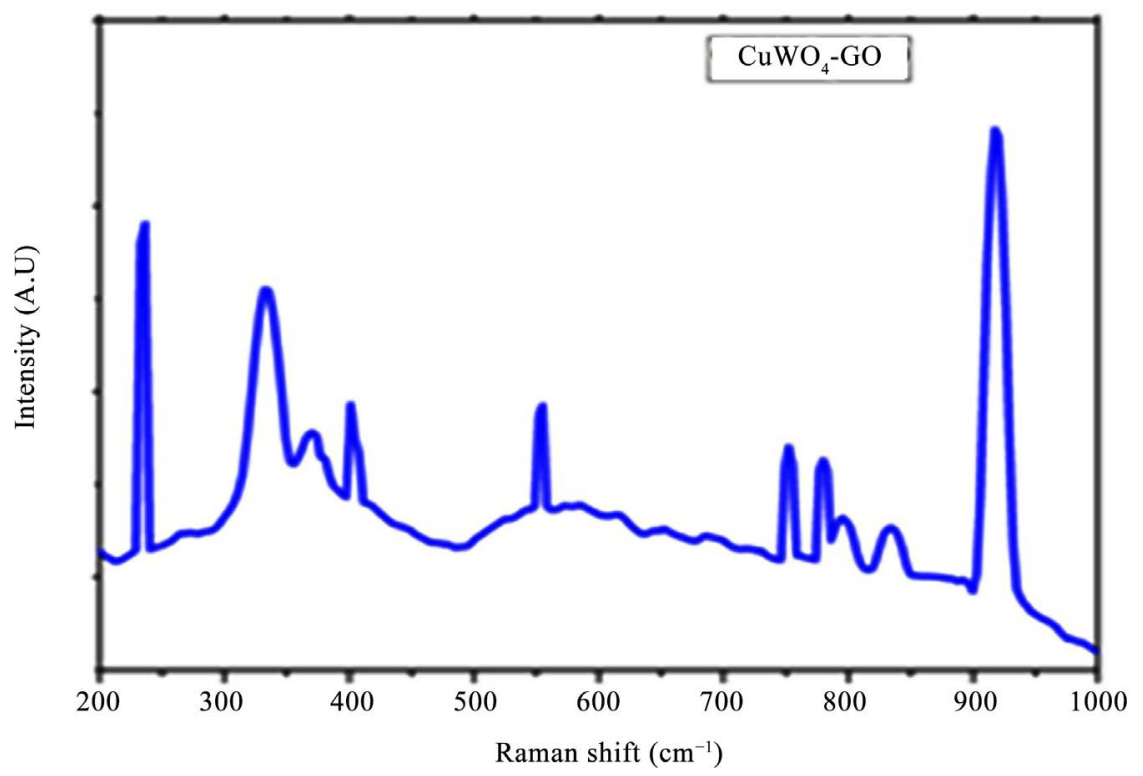

Figure 10. Raman spectra of $\mathrm{CuWO}_{4}$-GO nano composite.

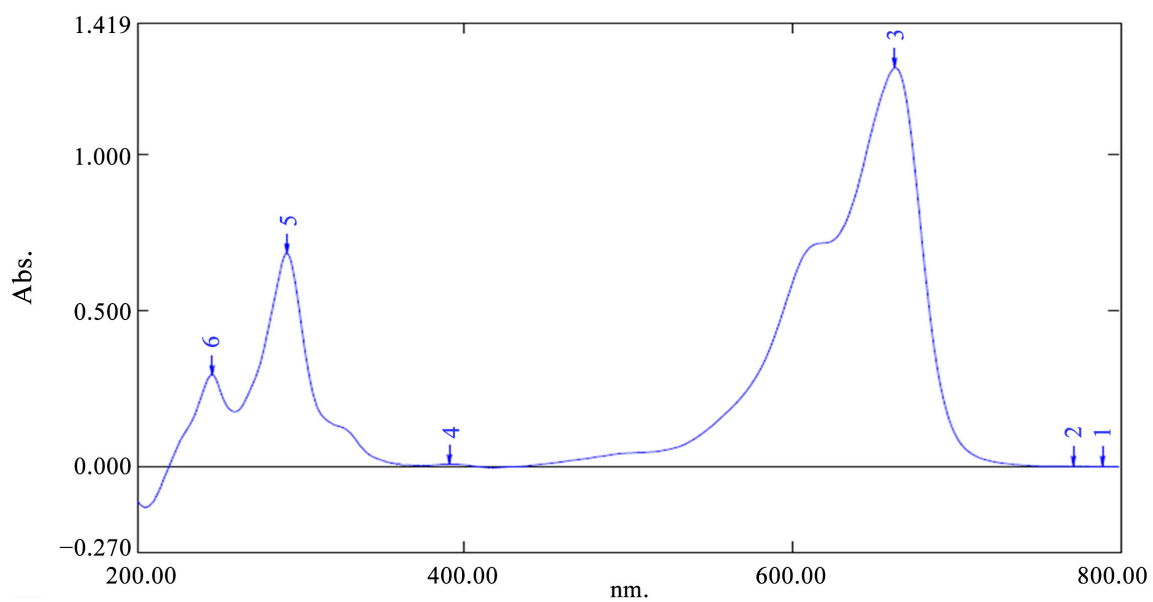

Figure 11. Absorption spectrum of methylene blue $\left(\lambda_{\max }\right.$ at $\left.667 \mathrm{~nm}\right)$.

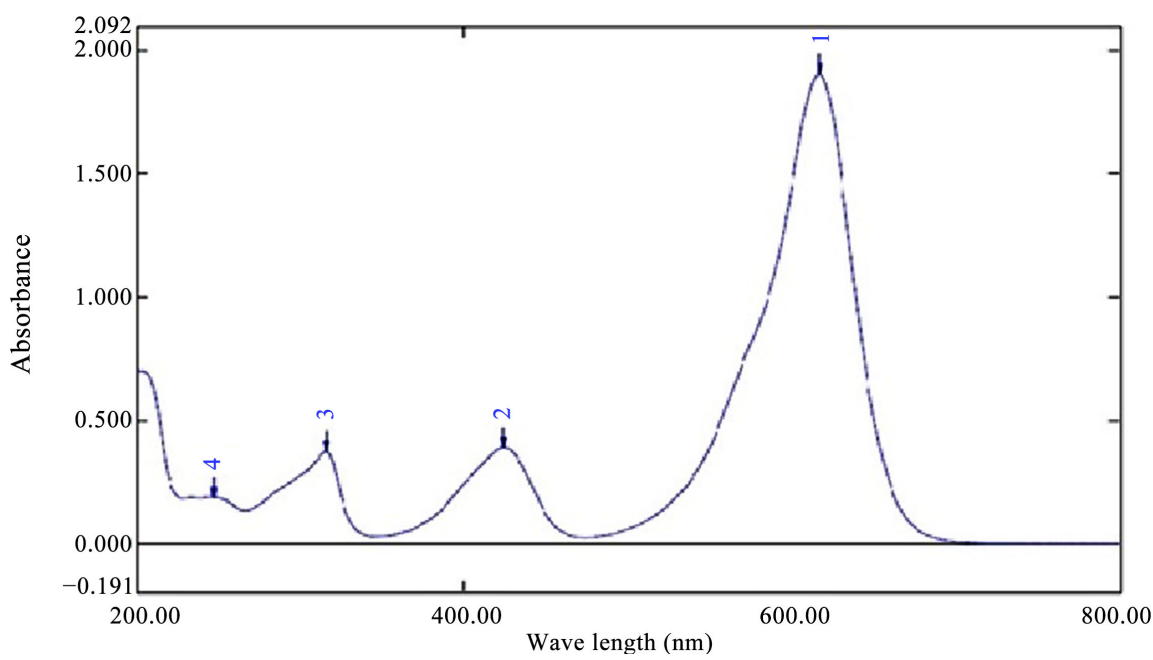

Figure 12. Absorption spectrum of malachite green $\left(\lambda_{\max } 617 \mathrm{~nm}\right)$. 


\subsection{The Photo Catalytic Activity}

The photo catalytic activity of the nanoCuWO${ }_{4}^{-}-\mathrm{GO}$ composite was investigated towards the degradation of organic dyes Methylene blue (MB) and Malachite green (MG) under visible light irradiation is represented in Figure 13 shown in comparison with micro and nanoCuWO 4 .

Aiming at eliminating the effect of adsorption on the dye degradation efficiency, before each photocatalytic process, the adsorption-desorption equilibria between the dye and the photocatalysts were first obtained at $30 \mathrm{~min}$ and it is sufficient time to reach equilibrium. After $30 \mathrm{~min}$ the reaction mixture is exposed to visible light under constant magnetic stirring, and aliquot of the reaction mixture is collected for every $10 \mathrm{~min}$ and subjected to UV-Vis analysis. It can be seen that the intensity of the absorption peaks decreased as the reaction progressed with $\mathrm{CuWO}_{4}$ - $\mathrm{GO}$ as the catalyst. We observed that the MG dye solution is completely degraded in $60 \mathrm{~min}$ and $\mathrm{MB}$ dye degraded in $80 \mathrm{~min}$.

\subsection{Effect of Graphene Oxide \% Composition on the Photocatalytic Activity}

Malachite green and Methylene blue dye can be more easily adsorbed by GO (MG\&MB degradation efficiency $~ 75 \%$ ) rather than the $\mathrm{CuWO}_{4}$ (MG \& MB degradation efficiency $\sim 3 \%$ ) catalyst. Its adsorption capacity was enhanced when $\mathrm{GO}$ was introduced on $\mathrm{CuWO}_{4}$. The performances of the prepared samples, including pure $\mathrm{CuWO}_{4}$ and modified $\mathrm{CuWO}_{4}$ by graphene oxide with different percent compositions, were investigated with regard to the degradation of MG\&MB under visible light irradiation. There is almost no photodegradation in the absence of the catalyst. This suggested that, negligible amount of degradation by light can be ignored. In the presence of nano $\mathrm{CuWO}_{4}$, Malachite green and Methylene blue dyes were degraded in 80 to100 min under visible light irradiation. The $\mathrm{CuWO}_{4}$-GO nano composite has enhanced the photocatalytic degradation of Malachite green and Methylene blue dyes. The efficiencies of dye degradation were significantly improved from $80 \%$ to $100 \%$ in a time interval of 60 to $80 \mathrm{~min}$ by improving the amount of graphene oxide from $5 \%$ to $10 \%$ in the $\mathrm{CuWO}_{4}$-GO composite. Even though by adding upto $20 \%$ of graphene oxide in the composite, degradation efficiency remained the same. This suggests that graphene oxide with an amount of $10 \%$ in the $\mathrm{CuWO}_{4}$ - $\mathrm{GO}$ composite performs the best in removing the organic pollutants in wastewater under visible light irradiation (Figure 14).

\subsection{Effect of Amount of $\mathrm{CuWO}_{4}$-GO Nano Composite}

The effect of photocatalysts ( $10 \% \mathrm{CuWO}_{4}$ - $\mathrm{GO}$ nano composite) dosage on the photocatalytic degradation of Malachite green and Methylene blue was studied and shown in Figure 15. The dosage amount was varied from $10 \mathrm{mg}$ to 100 $\mathrm{mg} / 100 \mathrm{~mL}$ and all the observations are carried out at room temperature. It can be concluded that, with the increase of catalyst dosage from $10 \mathrm{mg}$ to 50 $\mathrm{mg} / 100 \mathrm{ml}$, the degradation efficiencies were significantly improved. Further increase 


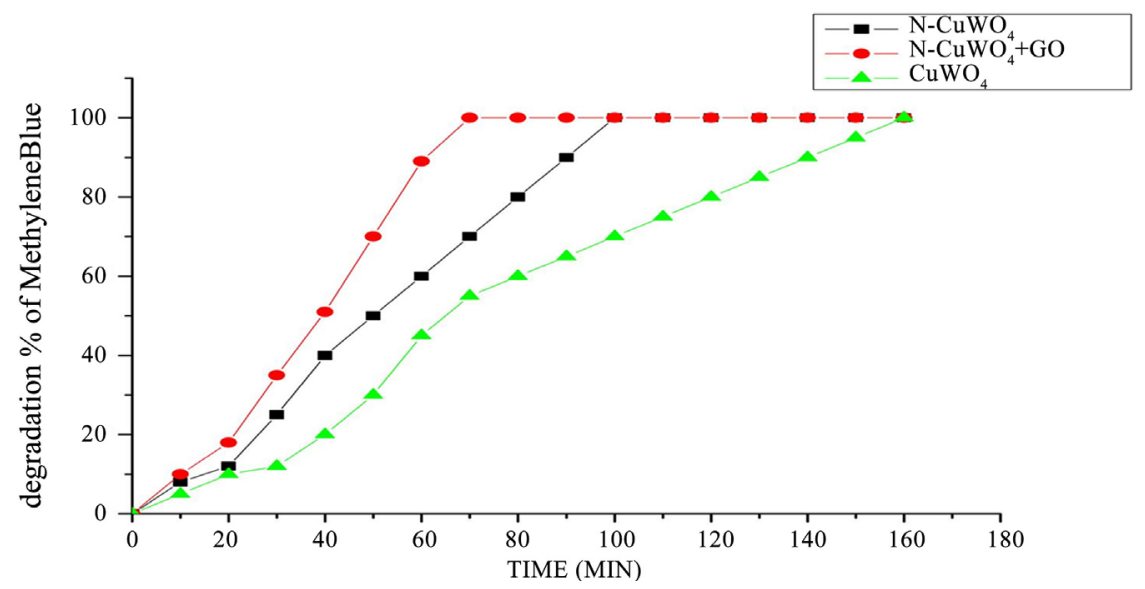

Figure 13. Comparative degradation of $\mathrm{CuWO}_{4}-\mathrm{GO}$ composite with micro and nano $\mathrm{CuWO}_{4}$.

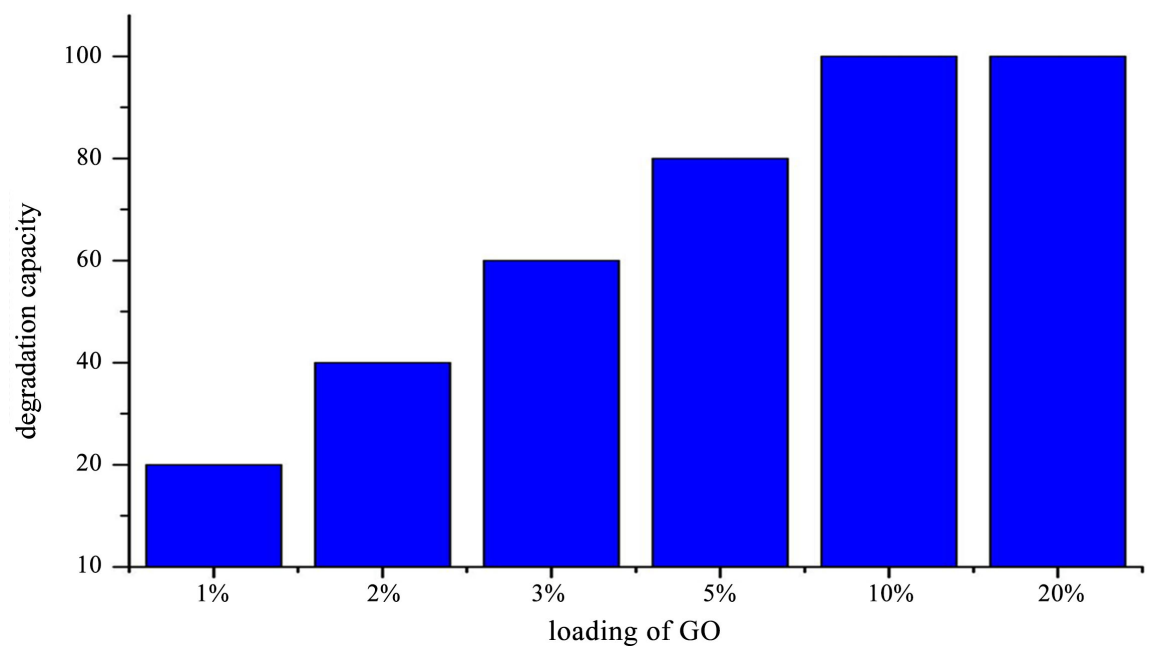

Figure 14. \% of GO composition on catalyst.

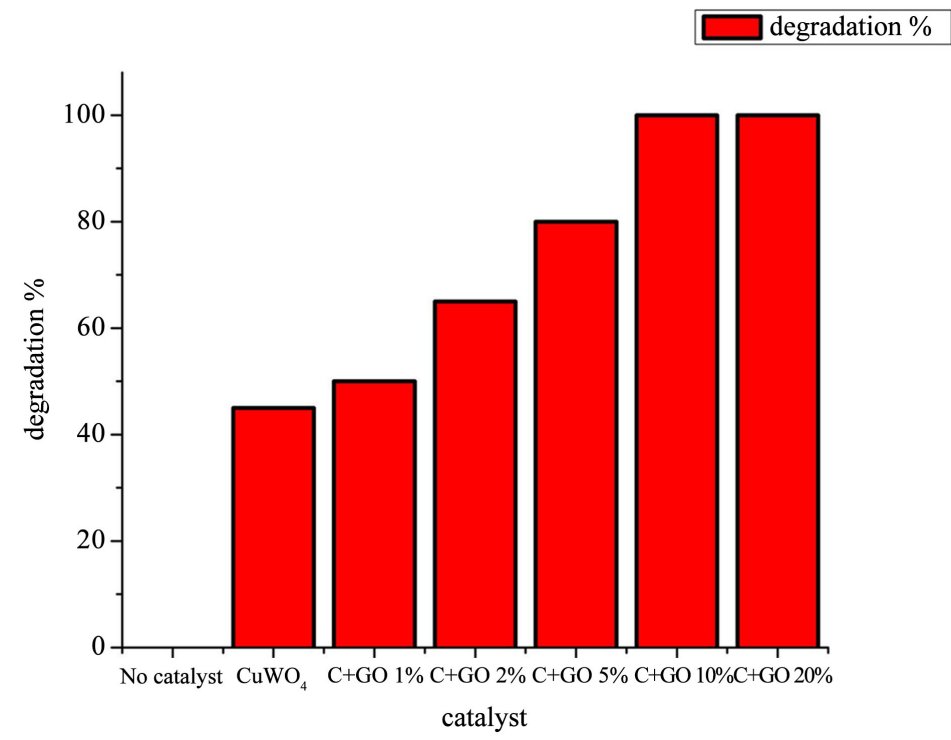

Figure 15. Effect of amount of $\mathrm{CuWO}_{4}$-GO nano composite. 
of the catalysts dosage quantity from 4 to $6 \mathrm{~g} / \mathrm{L}$ will slightly decrease the efficiency of the degradation of Malachite Green and Methylene blue. This phenomena, maybe because of the increase in the amount of catalysts dosage, which would increase the reactive sites that can correspondingly produce more reactive oxidative species. However, too much catalyst dispersed in the system will possibly increase light scattering and decrease light penetration [28], resulting in the reduction of degradation efficiency of $\mathrm{MG} \& \mathrm{MB}$ in a system with excessive photocatalysts. $\mathrm{CuWO}_{4}-10 \%$ and $\mathrm{CuWO}_{4}-20 \% \mathrm{GO}$ nano are having similar percentage of degradation so the experiment was carriedout with $\mathrm{CuWO}_{4}-10 \% \mathrm{GO}$ composite

\subsection{Effect of pH of Initial Dye Solution}

Considering that the $\mathrm{pH}$ of wastewater is possibly different, its effect on the photocatalytically degrading dye (MG/MB) in the presence of $\mathrm{CuWO}_{4}-\mathrm{GO}$ under visible light irradiation was explored. It has been reported in Figure 16 that, with an increase of $\mathrm{pH}$ of the dye (MG/MB) solution, it may reduce the adsorption of dye (MG/MB) on the photocatalyst. This resulted in the improvement of degradation efficiencies when $\mathrm{pH}$ of the MG solution increased from 5 to 7 and for $\mathrm{MB}$ solution increased from 3 to 5 [27]. And the transformation of $\mathrm{CuWO}_{4}$ damages the $\mathrm{CuWO}_{4}$-GO structure and eventually reduces the photocatalytic activity.

\subsection{Effect of Temperature}

As shown in Figure 17, temperature of the photocatalytic reacting system was also varied from $0^{\circ} \mathrm{C}$ to $80^{\circ} \mathrm{C}$ to explore its effect on the photocatalytic performances of the prepared samples under visible light irradiation. When the temperature of the reacting system is in the range of $20^{\circ} \mathrm{C}-60^{\circ} \mathrm{C}$, the photocatalytic performances in degrading dye (MG/MB) were similar and only a slight increase is found with the increase in temperature. However, when the temperature was fixed at $0^{\circ} \mathrm{C}$, the photocatalytic activity was significantly reduced. This might be because the mass transfer of pollutants to the surface of photocatalysts was decreased and the generation rate of oxidative species was also reduced. When the temperature was as high as $80^{\circ} \mathrm{C}$, the photocatalytic activity was greatly decreased. High temperature favors the recombination of charge carriers and desorption of adsorbed organics on the photocatalysts. The results can be regarded as evidence of temperature controller needed for solar devices.

\subsection{Reusability of the Catalyst}

The reusability of the prepared sample was assessed by recycling $\mathrm{CuWO}_{4}-\mathrm{GO}$ composite three times and the profiles of MG/MB concentrations is shown in Figure 18. The removal efficiency of dye (MG/MB) was $95 \%$ in the first run $60 \%$ in second run and 33\% in third run over 80 - 160 min under visible light irradiation. The slight decrease can be attributed to the loss of photocatalysts between two runs and some refractory intermediates adsorbed on their surface which are 

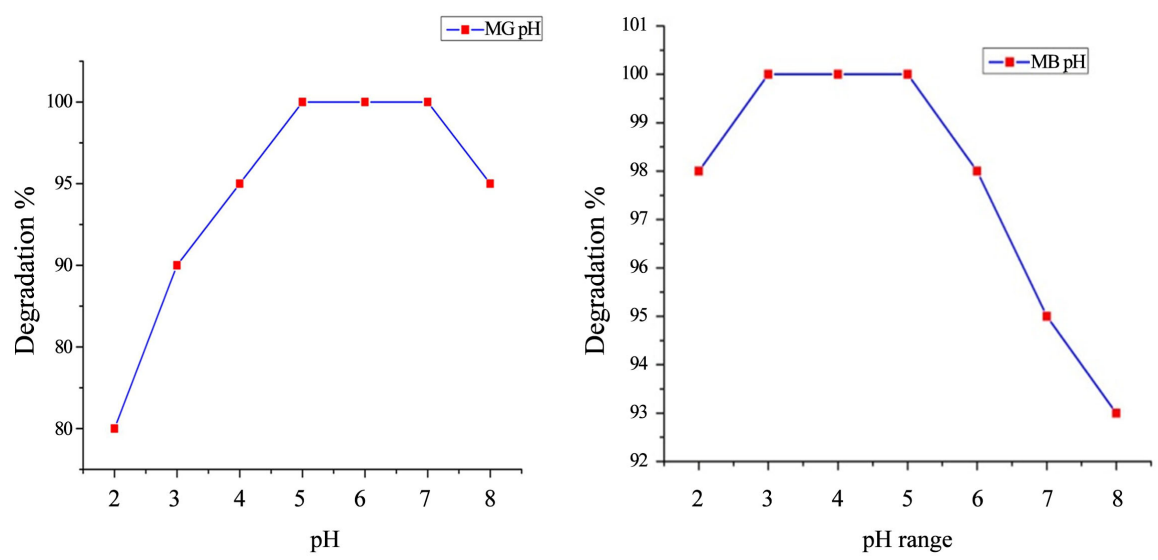

Figure 16. $\mathrm{pH}$ range of $\mathrm{CuWO}_{4}$-GO Nano composite on Malachite Green and Methylene Blue.
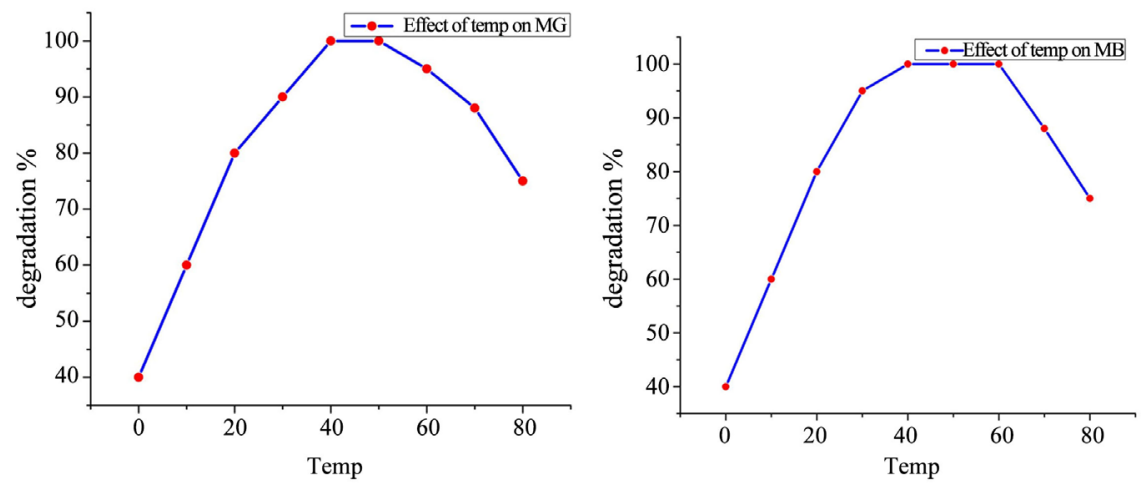

Figure 17. Temperature effect on $\mathrm{CuWO}_{4}$-GO Nano composite.
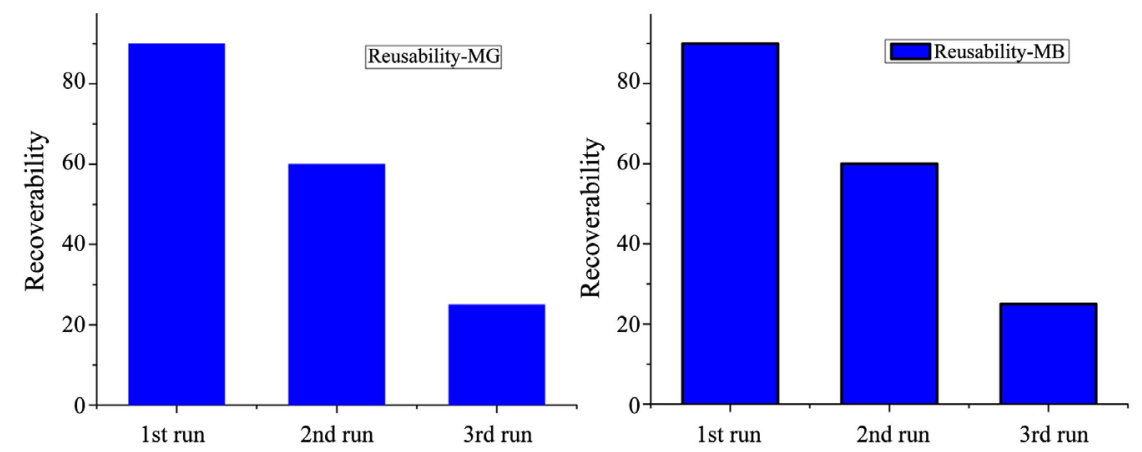

Figure 18. Reusable capacity of $\mathrm{CuWO}_{4}$-GO nano composite.

difficult to be destroyed [28]. Despite of this slight reduction in degradation efficiency, the stability of the reused $\mathrm{CuWO}_{4}$-GO photocatalysts after degradation of dye $(\mathrm{MG} / \mathrm{MB})$ is still significant.

\subsection{Plausible Photocatalytic Mechanism}

The plausible mechanism for the photocatalytic activity of the catalyst can be attributed to the presence of $\pi$-conjugation and $2 \mathrm{D}$ planar structures of graphene oxide in the $\mathrm{CuWO}_{4}$-GO composite which can adsorb organic molecules easily 
on its surface via strong $\pi-\pi$ interactions [29]. Additionally, graphene oxide possesses high charge carrier mobility and can be regarded as an electron acceptor. It can greatly decrease the recombination rate of photogenerated electrons and holes. Upon visible light excitation, the electron-hole pairs $\left(\mathrm{h}^{+}, \mathrm{e}^{-}\right)$are generated on the copper tungstate-graphene oxide surface followed by the instant transfer of photogenerated electrons onto graphene oxide via a percolation mechanism and then the negatively charged graphene oxide can activate the dissolved oxygen to produce superoxide anion radical, while the holes can react with the adsorbed water to form hydroxyl radical. Finally, the active species, holes, superoxide anion radical and hydroxyl radical oxidize the Methylene blue and Malachite green dye molecules adsorbed on the active sites of the copper tungstate-graphene oxide system through the $\pi-\pi$ stacking and electrostatic attraction.

$$
\begin{aligned}
& \mathrm{CuWO}_{4}-\mathrm{GO}+h v \rightarrow \mathrm{e}^{-}+\mathrm{h}^{+}+\mathrm{CuWO}_{4}-\mathrm{GO} \\
& \mathrm{h}^{+}+\mathrm{H}_{2} \mathrm{O} \rightarrow \cdot \mathrm{OH}+\mathrm{H}^{+} \\
& \mathrm{h}^{+}+\mathrm{O}^{-} \rightarrow{ }^{\bullet} \mathrm{OH} \\
& \mathrm{h}^{+}+\mathrm{C}_{16} \mathrm{H}_{18} \mathrm{~N}_{3} \mathrm{~S}^{+} \mathrm{Cl}^{-} \rightarrow\left(\mathrm{C}_{16} \mathrm{H}_{18} \mathrm{~N}_{3} \mathrm{~S}^{+} \mathrm{Cl}^{-}\right)^{+} \\
& \mathrm{h}^{+}+\mathrm{C}_{23} \mathrm{H}_{25} \mathrm{ClN}_{2} \rightarrow\left(\mathrm{C}_{23} \mathrm{H}_{25} \mathrm{ClN}_{2}\right)^{+} \\
& \mathrm{e}^{-}+\mathrm{O}_{2} \rightarrow{ }^{\bullet} \mathrm{O}_{2}^{-} \\
& \bullet \mathrm{O}_{2}^{-}+\mathrm{H}^{+} \rightarrow \cdot{ }^{\bullet} \mathrm{OOH} \\
& \cdot \mathrm{OOH} \rightarrow \mathrm{O}_{2}+\mathrm{H}_{2} \mathrm{O}_{2} \\
& \mathrm{H}_{2} \mathrm{O}_{2}+{ }^{\bullet} \mathrm{O}_{2}^{-} \rightarrow \cdot \mathrm{OH}+\mathrm{OH}^{-}+\mathrm{O}_{2} \\
& \mathrm{H}_{2} \mathrm{O}_{2}+h v \rightarrow 2^{\bullet} \mathrm{OH} \\
& \mathrm{C}_{16} \mathrm{H}_{18} \mathrm{~N}_{3} \mathrm{~S}^{+} \mathrm{Cl}^{-}+\left(\cdot \mathrm{OH}, \mathrm{H}^{+} \cdot \mathrm{OOH} \text { or } \mathrm{O}^{2-}\right) \rightarrow \mathrm{CO}_{2}+\mathrm{H}_{2} \mathrm{O}+\mathrm{SO}_{4}^{2-}+\mathrm{NO}_{3}^{-} \\
& \mathrm{C}_{23} \mathrm{H}_{25} \mathrm{ClN}_{2}+\left(\cdot \mathrm{OH}, \mathrm{H}^{+} \cdot \mathrm{OOH} \text { or } \mathrm{O}^{2-}\right) \rightarrow \mathrm{CO}_{2}+\mathrm{H}_{2} \mathrm{O}+\mathrm{SO}_{4}^{2-}+\mathrm{NO}_{3}^{-}
\end{aligned}
$$

\section{Conclusion}

In summary, we have demonstrated the synthesis of $\mathrm{CuWO}_{4}-\mathrm{GO}$ nano composite by metathesis and colloidal blending processes. The results demonstrated that $\mathrm{CuWO}_{4}-10 \%$ GO nano composite acts as an excellent photocatalyst for degradation of Methylene blue and Malachite green dyes under visible light irradiation. This catalyst causes complete degradation of Methylene blue dye in 60 min and Malachite green dye in 80 min of irradiation of visible light. The effect of $\mathrm{pH}$ and temperature on degradation and the reusability of the catalyst are also studied. As a result, this work provides a new method of developing a promising photo catalyst for the degradation of dyes.

\section{Acknowledgements}

Authors thank the UGC, SERO, Hyderabad for providing UGC-FDP Teacher 
Fellowship to M. Sunitha and UGC, MHRD, New Delhi for providing financial assistance through UGC-MRP No. 41-371/2012 (SR) to S. Paul Douglas. Authors thank Prof. M. Uma Bala, HOD, Dept. of Inorganic and Analytical Chemistry, Andhra University for UV-DRS studies, IICT Hyderabad for SEM, FT-IR and XRD, Centre for Nanotechnology, AUCE (A), AU for ball milling and JNTUK, Kakinada for Raman spectral studies.

\section{References}

[1] Honda, K. and Fujishima, A. (1972) Electrochemical Photolysis of Water at a Semiconductor Electrode. Nature, 238, 37-38. https://doi.org/10.1038/238037a0

[2] Michael, R., Hoffmann, Scot, T.M., Wonyong, C. and Detlef, W.B. (1995) Environmental Applications of Semiconductor. Photocatalysis Chem. Rev., 95, 69-96.

[3] Li, F.B., Li, X.Z. and Hou, M.F. (2004) Photo Catalytic Degradation of 2-Mercaptobiazole in Aqueous $\mathrm{La}^{3+}-\mathrm{TiO}_{2}$ Suspention for Odor Control. Applied Catalysis B, 48, 185-194. https://doi.org/10.1016/j.apcatb.2003.10.003

[4] Chamberland, B.L., Kafalas, J.A. and Goodenough, J.B. (1977) Characterization of Chromium Manganese Oxide $\left(\mathrm{MnCrO}_{3}\right)$ and Chromium(III) Manganate. Inorganic Chemistry, 16, 44-46.

[5] Gillette, R.H. (1950) Calcium and Cadmium Tungstate as Scintillation Counter Crystals for Gamma-Ray Detection. Review of Scientific Instruments, 21, 294-301. https://doi.org/10.1063/1.1745567

[6] Gopalakrishnan, J., Sivakumar, T., Ramesha, K., Thangadurai, V. and Subbanna, G.N. (2000) Transformations of Ruddlesden-Popper Oxides to New Layered Perovskite Oxides by Metathesis Reactions. Journal of the American Chemical Society, 122, 6237. https://doi.org/10.1021/ja9914644

[7] Sivakumar, T., Lofland, S.E., Ramanujachary, K.V., Ramesha, K., Subbanna, G.N. and Gopalakrishnan, J. (2004) Transforming $\mathrm{n}=1$ Members of the Ruddlesden-Popper Phases to a $\mathrm{n}=3$ Member through Metathesis: Synthesis of a New Layered Perovskite, $\mathrm{Ca}_{2} \mathrm{La}_{2} \mathrm{CuTi}_{2} \mathrm{O}_{10}$. Journal of Solid State Chemistry, 177, 2635. https://doi.org/10.1016/j.jssc.2004.03.030

[8] Mandal, T.K. and Gopalakrishnan, J. (2004) Fromrocksalt to Perovskite: A Metathesis Route for the Synthesis of Perovskite Oxides of Current Interest. Journal of Materials Chemistry, 14, 1273. https://doi.org/10.1039/b315263d

[9] Mani, R., Bhuvanesh, N.S.P., Ramanujachary, K.V, Green, W., Lofland, S.E. and Gopalakrishnan, J. (2007) A Novel One-Pot Metathesis Route for the Synthesis of Double Perovskites, $\mathrm{Ba}_{3} \mathrm{MM}_{2} \mathrm{O}_{9}\left(\mathrm{M}=\mathrm{Mg}, \mathrm{Ni}, \mathrm{Zn} ; \mathrm{M}^{\prime}=\mathrm{Nb}\right.$, Ta) with 1:2 Ordering of M and M' Atoms. Journal of Materials Chemistry, 17, 1589. https://doi.org/10.1039/B616238J

[10] Gillanand, E.G. and Kaner, R.B. (2001) Rapid, Energetic Metathesis Routes to Crystalline Metastable Phases of Zirconium and Hafnium Dioxide. Journal of Materials Chemistry, 11, 1951. https://doi.org/10.1039/b102234m

[11] Wiley, J.B., Gillan, E.G. and Kaner (1993) Rapid Soil State Metatheis Reactions for Te Synthesis of Copper of Oxide and Other Metal Oxides. Materials Research Bulletin, 28, 893. https://doi.org/10.1016/0025-5408(93)90035-C

[12] Ruiz-Fuertes, J., et al. (2010) High-Pressure Structural Phase Transitions in $\mathrm{CuWO}_{4}$. Physical Review B, 81, Article ID: 224115. https://doi.org/10.1103/PhysRevB.81.224115 
[13] Yourey, J.E. and Bartlett, B.M. (2011) Electrochemical Deposition and Photoelectrochemistry of $\mathrm{CuWO}_{4}$, a Promising Photoanode for Water Oxidation. Journal of Materials Chemistry, 21, 7651-7660. https://doi.org/10.1039/c1jm11259g

[14] Chang, Y., Braun, A., Deangelis, A., Kaneshiro, J. and Gaillard, N. (2011) Effect of Thermal Treatment on the Crystallographic, Surface Energetics, and Photoelectrochemical Properties of Reactively Cosputtered Copper Tungstate for Water Splitting. The Journal of Physical Chemistry, 115, 25490-25495.

[15] Paulchamy, B., Arthi, G. and Lignesh, B.D. (2015) A Simple Approach to Stepwise Synthesis of Graphene Oxide Nanomaterial. Journal of Nanomedicine and Nanotechnology, 6, 1

[16] Xu, Z. and Gao, C. (2011) Graphene Chiral Liquid Crystals and Macroscopic Assembled Fibers. Nature Communication, 2, Article No. 571.

https://doi.org/10.1038/ncomms1583

[17] Cong, H.P., Ren, X.C., Wang, P. and Yu, S.H. (2012) Wet-Spinning Assembly of Continuous, Neat and Macroscopic Graphene Fibers. Scientific Reports, 2, Article No. 613. https://doi.org/10.1038/srep00613

[18] Zhu, Y., Murali, S., Cai, W., Li, X., Suk, J.W., Potts, J.R. and Ruoff, R.S. (2010) Graphene and Graphene Oxide: Synthesis, Properties, and Applications. Advanced Materials, 22, 3906-3924. https://doi.org/10.1002/adma.201001068

[19] Du, X., Skachko, I., Barker, A. and Andrei, E. (2008) Approaching Ballistic Transport in Suspended Graphene. Nature Nanotechnology, 3, 491-495. https://doi.org/10.1038/nnano.2008.199

[20] Lee, C., Wei, X., Kysar, J.W. and Hone, J. (2008) Measurement of the Elastic Properties and Intrinsic Strength of Monolayer Graphene. Science, 321, 385-388. https://doi.org/10.1126/science.1157996

[21] Kim, J.E., Han, T.H., Lee, S.H., Kim, J.Y., Ahn, C.W., Yun, J.M. and Kim, S.O. (2011) Graphene Oxide Liquid Crystals. Angewandte Chemie International Edition, 50, 3043-3047. https://doi.org/10.1002/anie.201004692

[22] Ayrat, M.D. and James, M.T. (2014) Mechanism of Graphene Oxide Formation. ACS Nano, 8, 3060-3068. https://doi.org/10.1021/nn500606a

[23] Aliofkhazraei, M., Ali, N., Milne, W.I., Ozkan, C.S., Mitura, S. and Juana, L. (2016) Graphene Science Handbook: Fabrication Methods. CRC Press, Boca Raton.

[24] Wan, K.J. and Hyun, J.K. (2013) Titanium Dioxide-Graphene Oxide Composites with Different Ratios Supported by Pyrex Tube for Photocatalysis of Toxic Aromatic Vapors. Powder Technology, 250, 115-121. https://doi.org/10.1016/j.powtec.2013.10.017

[25] Chao, W., Ying, H., Xin, Z., Xuefang, C. and Jing, Y. (2016) Soft-Template Hydro Thermal Systhesis of Nanostructured Copper(II) Tungstate Cubes for Electrochemical Charge Storage Application. Electrochimica Acta, 220, 156-163.

https://doi.org/10.1016/j.electacta.2016.10.056

[26] Muthamizh, S., Suresh, R., Giribabu, K., Manigandan, R. and Praveen, S. (2014) Solid State Synthesis of Copper Tungstate Nanoparticles and Its Electrochemical Detection of 4-Chlorophenol. AIP Conference Proceedings, 1591, 508-510. https://doi.org/10.1063/1.4872655

[27] Hu, X., Meng, X. and Zhang, Z. (2016) Synthesis and Characterization of Graphene Oxide-Modified $\mathrm{Bi}_{2} \mathrm{WO}_{6}$ and Its Use as Photocatalyst. International Journal of Photoenergy, 10, 1155.

[28] Meng, X., Jiang, L., Wang, W. and Zhang, Z. (2015) Enhanced Photocatalytic Activ- 
ity of $\mathrm{BiOBr} / \mathrm{ZnO}$ Heterojunction Semiconductors Prepared by Facile Hydrothermal Method. International Journal of Photoenergy, 9, Article ID: 747024.

[29] Huang, H., Zhen, S., Li, P., Tzeng, S. and Chiang, H. (2016) Confined Migration of Induced Hot Electrons in $\mathrm{Ag} / g r a p h e n e / \mathrm{TiO}_{2}$ Composite Nanorods for Plasmonic Photocatalytic Reaction. Optics Express, 24, 15603-15608.

https://doi.org/10.1364/OE.24.015603 